\title{
Survival of post-treatment canine-to-canine lingual retainers with fiber-reinforced composite resin: a retrospective study
}

\author{
Davide Farronato, DDS, $\mathrm{PhD}^{1}$ \\ Roberto Briguglio, MD, DDS 2 \\ Francesco Mangano, DDS ${ }^{1}$ \\ Lorenzo Azzi, DDS ${ }^{3}$ \\ Giovanni Battista Grossi, MD, DDS ${ }^{3}$ \\ Francesco Briguglio, DDS, $\mathrm{PhD}^{2}$
}

${ }^{1}$ Department of Morphologic and Surgical Sciences,
Insubria University, Varese, Italy
2 Department of Experimental Sciences and Dentistry
Specialist Medical-Surgical, University of Messina,
Italy
${ }^{3}$ Department of Biomedical, Surgical and Dentistry
Science, University of Milan, Italy

Corresponding author:

Francesco Briguglio

Department of Experimental Sciences and Dentistry Specialist Medical-Surgical, University of Messina

Viale Gazzi

98100 Messina, Italy

E-mail: fra.briguglio@alice.it

\section{Summary}

The aim of the study is to evaluate the long term results of ribbond retainer after orthodontic treatment. One hundred and thirty patients who were orthodontically treated satisfied the inclusion criteria of having received a semipermanent retention were treated with FRC lingual retainers (Ribbond ${ }^{\circledR}$ ). It was performed a follow up evaluation after 2 years average from the retainer application and any complication or failure was recorded.

Data from 119 remaining patients that met the inclusion criteria were analyzed and no instances of loosening were observed. It may be concluded that orthodontic canine-to-canine FRC retainers provide aneffective means of retaining realigned anterior teeth for at least two years.

Key words: retention, ribbond, orthodontic treatment, FRC, movement of relapsing.

\section{Introduction}

Retention is the phase of orthodontic treatment that attempts to maintain teeth in their corrected positions after active tooth movement. The etiology of relapse is not ful- ly understood, but relates to a number of factors, including periodontal and occlusal factors, soft tissue pressures and growth (1). This has led many orthodontists to conclude that permanent retention may be the only reliable way to keep ideal alignment after treatment (2-9). Many appliance types have been used for the retention of posttreatment tooth position, both fixed (10) and removable (11). In order for retainers to be acceptable they must maintain the teeth in position without compromising oral health. They must also be acceptable to patients and be reliable (12).

At the present, two types of fixed retainers are in use: a heavier wire bonded only on the canines and a lighter wire, usually multi-stranded, bonded to both the incisors and the canines (13-15).

As an alternative to multistrand wire, the use of fiber-reinforced composite (FRC) retainers has been developed (16-20). These FRC are available in varying widths and forms, including braided polyethylene fibers (Connect ${ }^{\circledR}$, Kerr Inc, Orange, CA, USA), woven polyethylene fibers (Ribbond ${ }^{\circledR}$, Ribbond Inc., Seattle WA, USA) as well as other fiber types and forms. The clinicians may use strips of reinforcing fiber bundles that already have been impregnated with resin in a prior controlled manufacturing process or may incorporate a lowviscosity dental resin into the reinforcement at chairside. Whether the FRC is prepared by the dentist or purchased already impregnated, it is usually applied in conjunction with a restorative composite in the fabrication of a two component fixed splint. The FRC material is used as a substructure to provide increased strength and rigidity beneath an outer layer of particulate composite, which provides an aesthetic appearance and better wear resistance (21).

Several studies have assessed the effectiveness of different types of bonded wire retainers (22-30), showing a large range in failure rates. Unfortunately, limited clinical information is available concerning the reliability of FRC retainers for long term retention of mandibular and maxillary anterior segment from canine-to-canine after orthodontic treatment $(12,31,32)$.

Therefore, the aims of this study were to retrospectively evaluate the clinical survival rate of FRC lingual retainers by means of a historic cohort study, and to investigate the influence of gender, patient age and retainer location on survival.

\section{Materials and methods}

\section{Sample}

The records of individuals participating in this study 
were selected from a larger pool of patients treated at the practice of the first author (D.F.).

One hundred and thirty patients who were orthodontically treated satisfied the inclusion criteria of having received a semipermanent retention between January 2004 and July 2006. All identical type of FRC lingual retainers (2 $\mathrm{mm}$ woven polyethylene ribbon-reinforced; Ribbond ${ }^{\circledR}$, Ribbond Inc, Seattle WA, USA) (Fig. 1) were bonded from canine to canine in the maxilla, mandible or in both arches, using the same procedures with the same materials, by a single operator. Periodontally unhealthy teeth were excluded as fmps and fmbs was not lower than $20 \%$ or if any tooth was mobile or prosthetically restored. Composite restorations were accepted in inclusion criteria but in those cases sandblasting was performed under dam to increase the retainer adhesion.

\section{Application of retainers}

Routine adhesive removal and polishing were performed (33) and instruction in oral hygiene were carefully carried out before FRC retainers were inserted. The technique for placing the FRC retainer included placement of the dental dam extending to the first premolars bilaterally. Tooth surfaces from canine to canine were etched with a $37 \%$ orthophosphoric acid gel for 30 seconds, then rinsed with an oil-free air-water spray for 40 seconds and left slightly moist for the wet bonding technique. An adhesive system (Clearfil Se Bond, Kuraray America, Inc. New York, NY, USA) was applied following the manufacturer's instructions, using a disposable brush and including the interproximal surfaces and facial areas. The resin was not light cured at this time. Five $30-\mathrm{cm}$ pieces of dental floss (Crest Glide, Procter \& Gamble, Cincinnati, Ohio USA) were cut and tied all together at one end. Keeping the knot on the facial side, the free ends of the floss were threaded into inter-canine interproximal spaces and pulled lingually. A thin layer of flowable composite (Enamel Plus HFO Flow, Micerium, Avegno GE Italy) was placed onto the lingual surface from canine-to-canine and light cured for 20 seconds. The dental arch length from canine to canine was deter-

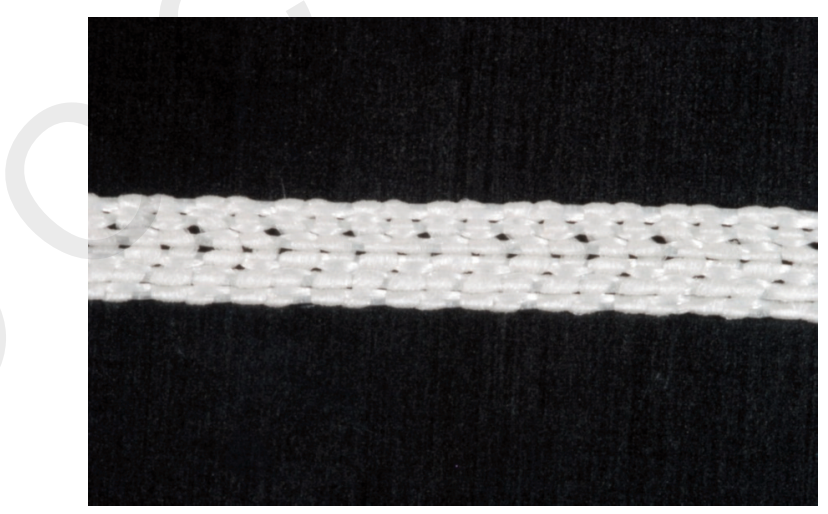

Figure 1. Two mm wide Ribbond ${ }^{\circledR}$ used as orthodontic lingual retainer. mined by closely adapting a piece of tinfoil to the lingual festooning of the teeth (Fig. 2). The required length of Ribbond was cut with a special scissors (Ribbond fiber cutter, Ribbond Inc., Seattle WA, USA) supplied by the manufacturer to prevent unraveling. The fiber ribbon was saturated with a few drops of bonding agent (Clearfil Se Bond, Kuraray America, Inc. New York, NY, USA) and embedded with flow (Enamel Plus HFO Flow, Micerium, Avegno GE Italy). Care was taken to keep the wet ribbon away from light to prevent initial polymerization, which would interfere with its manipulation. The wetted Ribbond was placed and the flosses were bent in an upward direction to position the FRC just above the interproximal areas. The Ribbond was closely adapted into the interproximal contacts against the teeth by the help of instruments and light cured for 40 seconds from lingual and proximal directions. Then, flosses were removed and fibers were coated approximately $0,5 \mathrm{~mm}$ with another layer of light-cured composite (Enamel plus HFO, Micerium, Avegno GE Italy) and the embrasures were shaped in order to facilitate good oral hygiene, paying attention not to have any part of the fiber exposed (Fig. 3). Excess resin was removed and a thin application of glycerin was placed on all the retainer to pre-

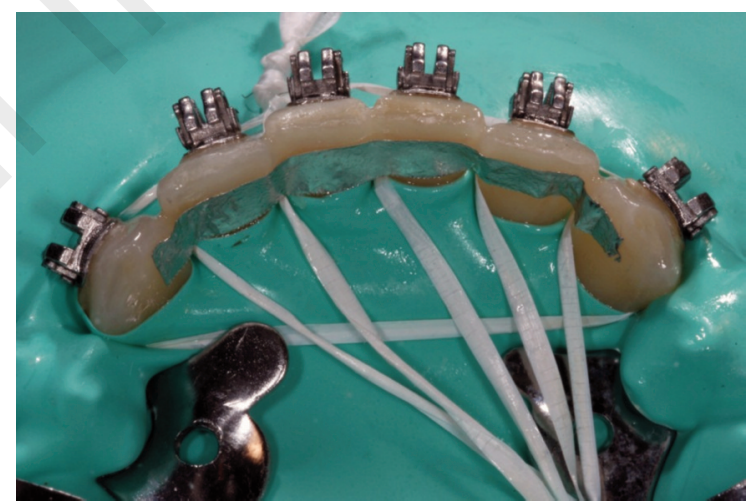

Figure 2. Placing flosses into inter-canine interproximal spaces and determination of the required length of ribbon by adapting a piece of tinfoil to the lingual surface of the teeth.

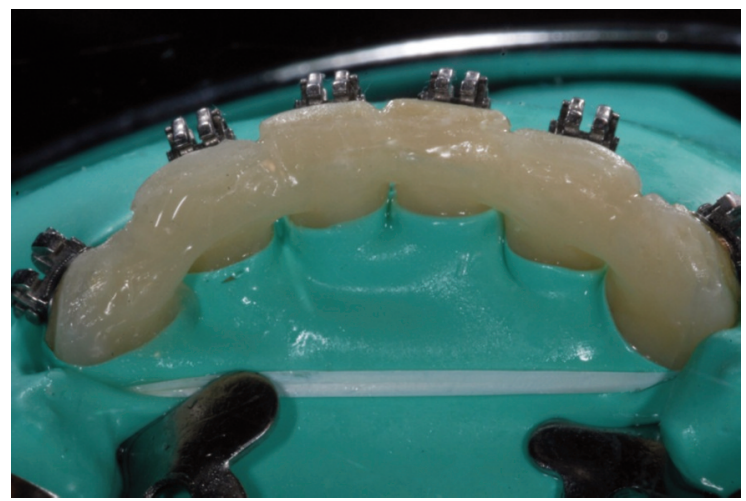

Figure 3. The lower FRC retainer in place. 
vent the formation of an oxygen-inhibiting layer on the resin. The FRC retainer was subsequently polymerized from all aspects (e.g. facial, incisal, lingual, proximal) for 60 seconds, respectively. Finally, after removal of the rubber-dam, the occlusion was checked with the use of a 32- $\mu \mathrm{m}$ articulation paper and adjusted if necessary.

Every patient was clearly informed of the importance of oral hygiene by giving more attention to plaque control and was instructed to use dental floss for proper interproximal oral hygiene.

\section{Follow-up and definition of clinical events}

Average follow up was 39.9 months (median 40.7 months, IQR 14.9, months, SD 13.3 months). After bonding, the patients were recalled for clinical examinations every 6 months, or when the patients reported a failure. During the follow-up period all interventions were recorded, such as polishing and finishing after chipping of small fragments of the resin composite, repair of small delaminations with restorative resin composite, or rebonding of $\mathrm{FRC}$ retainer after loosening.

If the FRC retainers debonded were lost during the follow-up period, they were registered as "absolute failure". When less severe clinical events occurred, such as fracture or delamination of the composite without loosening of the bond between composite and the teeth bonded, the $\mathrm{FRC}$ retainers were repaired and the event was registered as "relative failure" (Fig. 4a, b).

The wearing time was measured in months and was calculated as the time between bonding and any clinical event recorded as failure. Only the first failures were counted and a reported failure in the maxilla or in the mandible was counted as a separate incidence. In addition, multiple failure sites in one retainer were counted as one failure. All data were recorded by being typed directly into an anonymized database.

\section{Statistical analysis}

Statistical analysis was performed using the Statistical Package for Social Sciences (version 18.0, SPSS Inc., Chicago, Illinois, USA). Descriptive statistics and Kaplan-Meier curves were calculated.

Survival was defined at two levels: functional survival (endpoints: absolute failures) and overall survival (endpoints: both absolute and relative failures). Observations with no event in the respective analysis were censored at the end of the individual observation period. Kaplan-Meier survival analyses were done for the complete group of FRC retainers and discriminated according to gender, age of the groups and retainer location.

\section{Results}

Of 130 patients, eleven were not able or willing to be clinically evaluated. Therefore, data from 119 remaining patients that met the inclusion criteria were included in the analysis. Of these, 15 patients had FRC retainers in both arches, so that a total of 134 FCR retainers were studied.

The maximum follow-up period was 61.4 months (median 40.7 months, IQR 14.9 , mean 39.9 months, SD 13.3). Most of the patients were adults, with a median age of 32.5 years at the time of follow-up examination. No instances of loosening were observed, therefore the functional survival rate was $100 \%$. Table 1 shows a summary of the demographic characteristics of the patient population and the effect of confounding factors on the overall survival rate. In total, relative failure (fracture or delamination of the composite) was recorded in 25 FRC retainers, corresponding to $18.7 \%$ of the 134 retainers bonded. Conversely, the present study shows that the FRC location exerted a significant influence on the failure rate. In fact, the incidence of relative failures was remarkably lower for the mandibular than for the maxillary FRC retainers.
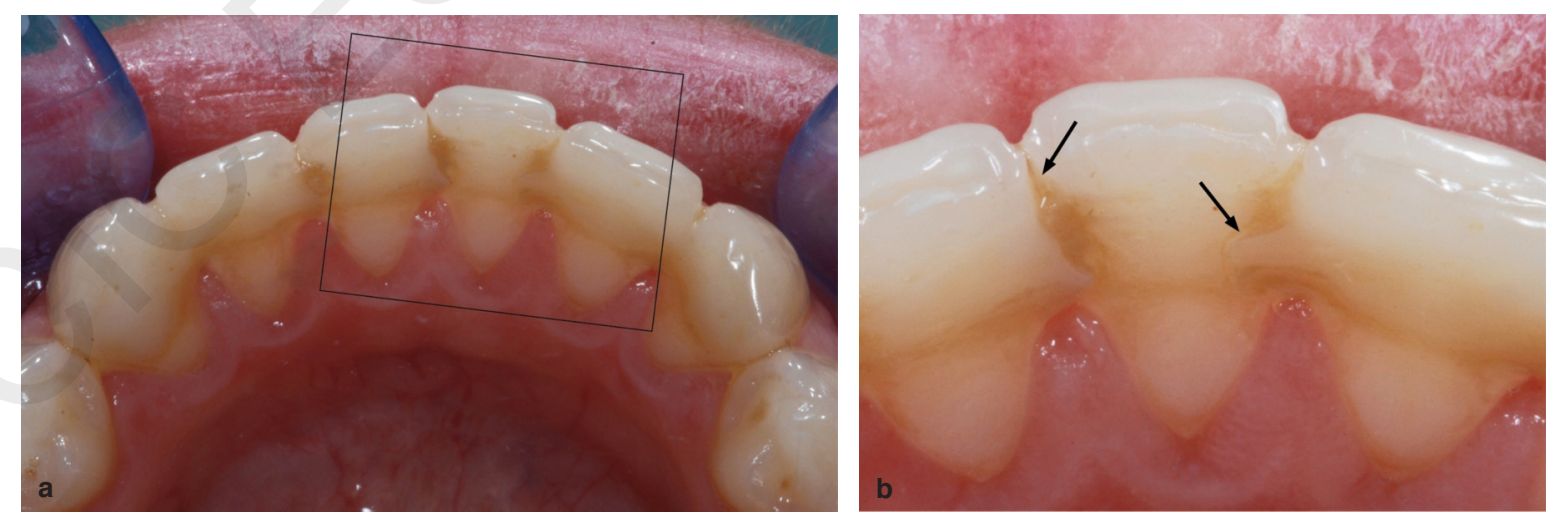

Figure 4. (a) FRC retainer's relative failure at 60 months' follow-up. (b) Higher magnification of the retainer, showing interproximal delamination and fracture of the composite (arrows). Is possible to notice plaque accumulation in correspondence to the gap, the interproximal wire can't pass between teeth and demonstrate the fiber integrity. In this condition fiber keepon helding the fractured parts together avoiding teeth migration (Pekka K. Vallittu: Ultra-high-modulus polyethylene ribbon as reinforcement for denture polymethyl methacrylate: A short communication, Dent Mater 13:381-382, November,1997), but there could be high risk of infiltrating caries in the interproximal area and unaesthetic discoloration. 
Figure 5 shows that the cumulative overall survival rate for the FRC retainers was $81.3 \%$. Kaplan-Meier overall survival rates at 5 years were higher for mandibular retainers $(86.3 \%)$ compared to maxillary retainers $(65.6 \%)$. These differences in survival percentages were statistically different (Fig. 6, log rank test, $\mathrm{P}=0.007$ ).

\section{Discussion}

Orthodontic canine-to-canine retainers are frequently used to avoid relapse and secondary crowding of incisors. Such retainers may either be fixed or removable. Fixed lingual retainers were introduced in the 1970s (10) and are often made of stainless steel wires. The major advantage of bonded canine-to-canine retainers relative to removable is that they are compliance free. However, if the retainer wire is not

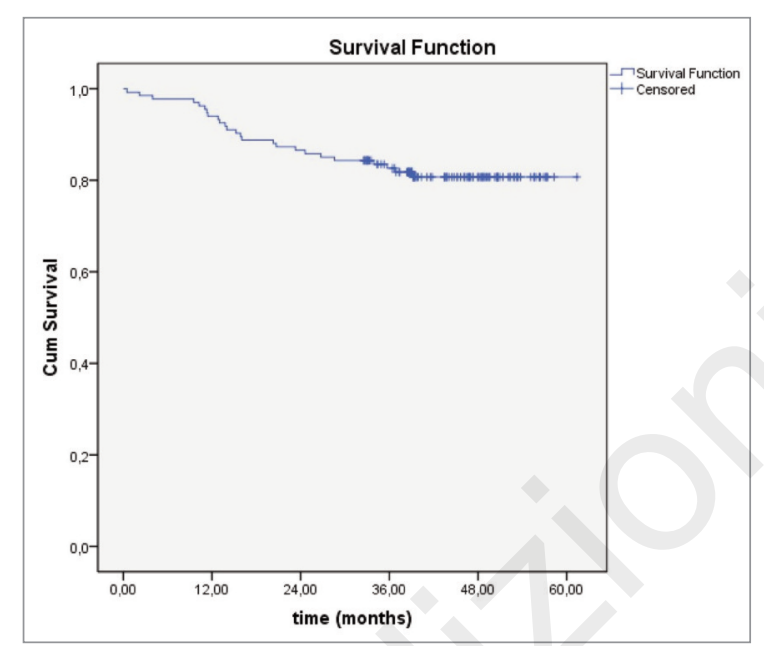

Figure 5. Kaplan-Meier survival curve showing a $81.3 \%$ success rate for the FRC retainers over a 61.4 month period.

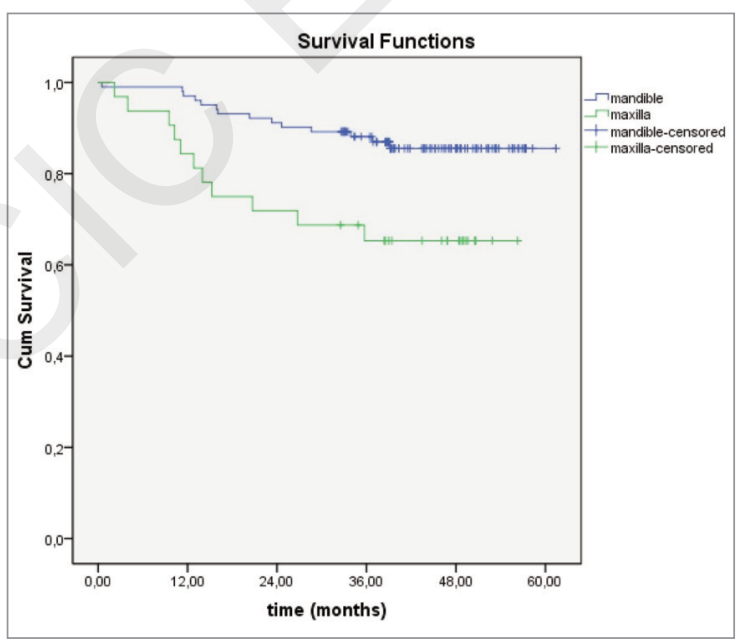

Figure 6. Survival percentages. passive at the time of bonding, the teeth may move. Another technique-related problem is frequent bond failure, either in the wire/composite interface if too little composite is added, or in the adhesive/enamel interface in situations with moisture contamination or retainer movement during the bonding procedure (34). In addition to bond failure, stress fracture of the interdental wire segments was reported (22). Some clinical studies have shown that there is a relatively high failure rate ranging between 10.3 to $47 \%$, depending on the technique used and follow-up observation period $(22,27-29,35)$.

Recently, FRC materials have been introduced for the fabrication of fixed dental prostheses, root posts and periodontal splints and much research is currently being conducted for these clinical applications (3643). Conversely, very few studies have focused on the use of FRCs as orthodontic retainers $(31,44,45)$. A main advantage of a $\mathrm{FRC}$ retainer compared with the classic twist-flex retainer is its high transparency, resulting in an almost invisible retainer (Fig. 7a-c). The retainer can thus be placed close to incisal edge. This is an advantage from both biological and biomechanical points of view (44). One major disadvantage of the $\mathrm{FRC}$ retainer is that the placement procedure is time consuming and technique sensitive. Moreover, it is more expensive than other types of bonded retainers.

To the best of our knowledge, this is the first large investigation to have analyzed the clinical survival rate of FRC lingual retainers for a long follow-up period. Of the 134 orthodontic retainers being followed none have exhibited debonding and the only kind of failure was fracture or delamination of the interproximal composite $(18.7 \%)$ without loosening of the bond between composite and the 804 teeth bonded. Therefore, $100 \%$ of the retainers were still in place after 5 years.

These results are substantially different from those obtained in the clinical trial by Rose et al. (36), in which a total of 20 patients were assigned to receive Ribbond ${ }^{\circledR}$ fiber or multistranded wire canine-to-canine retainers. In terms of survival time, the results reported by Rose would suggest that the multistranded wire is significantly superior to the FRC retainer. It should, however, be noted that their sample size was only 10 for each group and no sample size calculation were reported. Moreover, the protocol described in the Rose's article would be counter to the protocols used for the construction of any fiber-reinforced structure (44, 46-48). In fact, the authors polymerized the composite before applying the fiber and did not adapt the fiber closely against the teeth. Conversely, in the current study the manufacturer's instructions were followed (14). In order for fiber to work, the resin composite was not cured until after the fiber was closely adapted against the tooth surface. In fact, placing the fiber against the surface minimizes the bond line thickness between the teeth and the fiber and, just like orthodontic brackets, the thinner the layer of bonding resin composite between the teeth and the laminate, the better the results. Whichever reinforcement technique is used, it is critical that manufactur- 

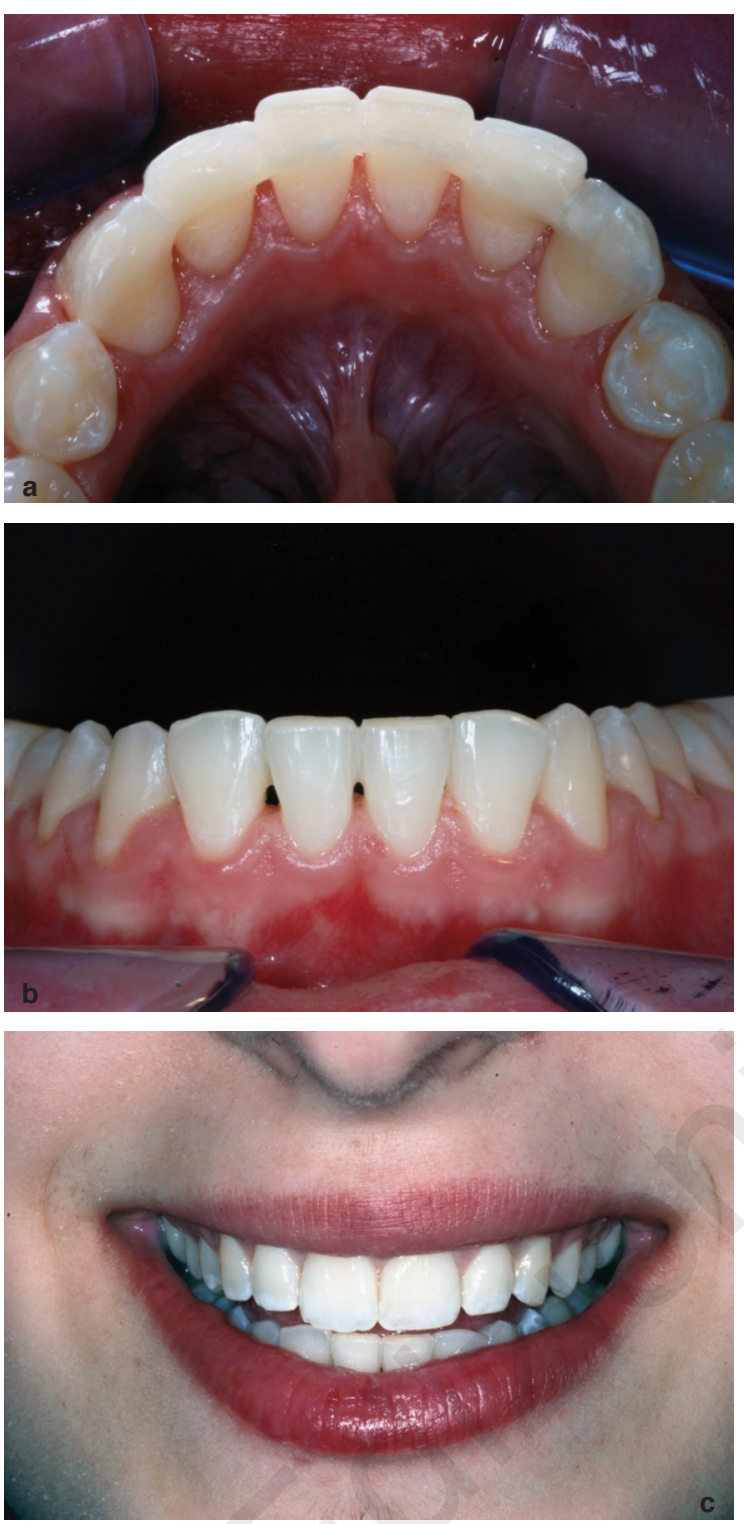

Figure 7. (a) Mandibular FRC retainerat five-year follow-up showing $(b, c)$ good aesthetics.

er's instructions be followed. These reinforcement materials are technique sensitive, meaning that the material does not perform well if its method of use is left to uninformed technical application or random chance (14).

The principal reason given by Rose et al. (31) for the reduced reliability of the ribbon-reinforced retainers was that FRC materials would possess greater yield strength than the wire, holding the teeth in a rigid manner and resulting in more likely debonding. Foek et al. (45) recently compared the bond strength of various stainless orthodontic wires vs various FRC retainers and found no significant differences between the wire groups and the Ribbond ${ }^{\circledR}$ retainers. Interestingly, the failure rates recorded in the present investigation are also more favorable than those published previously for stainless steel wires $(1,35)$. One explanation for this could be that all FRC retainers were applied by only one operator in our study, using the same materials and the same bonding techniques under rubber dam isolation. In fact, the bonding procedures are technique sensitive and factors such as minute movements of the retainer during the setting process of the adhesive or a lack of moisture control (49) could impair ideal adhesion. Dahl and Zachrisson's $(14,15)$ reported the lower failure rates $(9.8 \%$ of loosening and wire fracture over a 38-month-observation period) with the use of the five-stranded spiral wire retainers (Penta-One), bonded by the same orthodontist. In a long-term clinical study where the retainers were bonded by 15 different operators Foek et al. (23) reported a failure rate of $37.9 \%$. Interestingly, however, they found that neither different operators nor experience played a significant role in failure rate.

Finally, our data showed no significant differences in failure rates between genders and age, in agreement with the findings of previous studies $(23,24)$. Moreover, in keeping with other studies $(22,24,50,51)$, there is clear evidence that FRC retainers in the upper arch are much more likely to fail than in the lower arch, and this may reflect the role of occlusal factors in the failure of these retainers (29).

\section{Conclusions}

It may be concluded that orthodontic canine-to-canine FRC retainers provide an effective means of retaining realigned anterior teeth for at least five years.

Careful preparation and adaptation of the Ribbond ${ }^{\circledR}$ fiber along with strict moisture control are the critical steps for success in the FRC retainers.

Nevertheless, there is a need for further controlled studies, both in vitro and in vivo, to validate the findings of this investigation.

\section{References}

1. Melrose C, Millett DT. Toward a perspective on orthodontic retention? Am J Orthod Dentofacial Orthop. 1998;113:507-514.

2. Littlewood SJ, Millett DT, Doubleday B, Bearn DR, Worthington HV. Orthodontic retention: a systematic review. J Orthod. 2006 Sep;33(3):205-12.

3. Cerny R. The reliability of bonded lingual retainers. Aust Orthod J. 2007;23:24-29.

4. Durbin DD. Relapse and the need for permanent fixed retention. J Clin Orthod. 2001; 35:723-727.

5. Salehi $P$, Zarif Najafi H, Roeinpeikar SM. Comparison of survival time between two types of orthodontic fixed retainer: a prospective randomized clinical trial. Prog Orthod. 2013 Sep 11:14:25.

6. Lassaire J, Costi A, Charpentier E, Castro M. Post-orthodontic intra- and interarch changes at 1 year: a retrospective study assessing the impact of anterior fixed retention. Int Orthod. 2012 Jun;10(2):165-76.

7. Stability and relapse of dental arch alignment. Little $\mathrm{RM} . \mathrm{Br}$ J Orthod. 1990 Aug;17(3):235-41.

8. Nanda RS, Nanda SK. Considerations of dentofacial growth in long-term retention and stability: is active retention needed? Am J Orthod Dentofacial Orthop. 1992;101:297-302. 
9. Parker WS. Retention-retainers may be forever. Am J Orthod Dentofacial Orthop. 1989;95:505-513.

10. Butler J, Dowling P. Orthodontic bonded retainers. J Ir Dent Assoc. 2005 Spring;51(1):29-32.

11. Hawley CA. A removable retainer. Int J Orthod. 1919;2:291298.

12. Littlewood SJ, Millett DT, Doubleday B, Bearn DR, Worthington HV. Orthodontic retention: a systematic review. J Orthod. 2006;33:205-212.

13. Littlewood SJ, Millett DT, Doubleday B, Bearn DR, Worthington HV. Retention procedures for stabilising tooth position after treatment with orthodontic braces. Cochrane Database Syst Rev. 2006:CD002283.

14. Zachrisson BU. The bonded lingual retainer and multiple spacing of anterior teeth. Swed Dent J Suppl. 1982;15:247-255.

15. Zachrisson BU. Long-term experience with direct-bonded retainers: update and clinical advice. J Clin Orthod. 2007;41:728737; quiz 749 .

16. Wu HM, Zhang JJ, Pan J, Chen D. Clinical evaluation of glass fiber-reinforced composites for fixed orthodontic lingual retainers. Shanghai Kou Qiang Yi Xue. 2014 Feb;23(1):80-2.

17. Freudenthaler JW, Tischler GK, Burstone CJ. Bond strength of fiber-reinforced composite bars for orthodontic attachment. Am J Orthod Dentofacial Orthop. 2001;120:648-653.

18. Geserick M, Ball J, Wichelhaus A. Bonding fiber-reinforced lingual retainers with color-reactivating flowable composite. J Clin Orthod. 2004;38:560-562.

19. Goldberg AJ, Freilich MA. An innovative pre-impregnated glass fiber for reinforcing composites. Dent Clin North Am. 1999;43:127-133, vi-vii.

20. Orchin JD. Permanent lingual bonded retainer. J Clin Orthod. 1990;24:229-231.

21. Strassler HE, Serio CL. Esthetic considerations when splinting with fiber-reinforced composites. Dent Clin North Am. 2007;51:507-524; xi.

22. Andren A, Asplund J, Azarmidohkt E, Svensson R, Varde P, Mohlin B. A clinical evaluation of long term retention with bonded retainers made from multi-strand wires. Swed Dent J. 1998;22:123-131.

23. Artun J, Spadafora AT, Shapiro PA. A 3-year follow-up study of various types of orthodontic canine-to-canine retainers. Eur J Orthod. 1997;19:501-509.

24. Ye L, Yang P. Bonded lingual retainers. Am J Orthod Dentofacial Orthop. 2013 May;143(5):596.

25. Booth FA, Edelman JM, Proffit WR. Twenty-year follow-up of patients with permanently bonded mandibular canine-tocanine retainers. Am J Orthod Dentofacial Orthop. 2008;133:70-76.

26. Bolla E, Cozzani M, Doldo T, Fontana M. Failure evaluation after a 6-year retention period: a comparison between glass fiber-reinforced (GFR) and multistranded bonded retainers. Int Orthod. 2012 Mar;10(1):16-28.

27. Lie Sam Foek DJ, Ozcan M, Verkerke GJ, Sandham A, Dijkstra PU. Survival of flexible, braided, bonded stainless steel lingual retainers: a historic cohort study. Eur J Orthod. 2008;30:199-204.

28. Lumsden KW, Saidler G, McColl JH. Breakage incidence with direct-bonded lingual retainers. Br J Orthod. 1999;26:191-194.

29. Renkema AM, Al-Assad S, Bronkhorst E, Weindel S, Katsaros C, Lisson JA. Effectiveness of lingual retainers bonded to the canines in preventing mandibular incisor relapse. Am J Orthod Dentofacial Orthop. 2008;134:179-180.

30. Stormann I, Ehmer U. A prospective randomized study of different retainer types. J Orofac Orthop. 2002;63:42-50.

31. Rose E, Frucht S, Jonas IE. Clinical comparison of a multistranded wire and a direct-bonded polyethylene ribbon-reinforced resin composite used for lingual retention. Quintessence Int. 2002;33:579-583.
32. Cordasco G, Farronato G, Festa F, Nucera R, Parazzoli E, Grossi GB. In vitro evaluation of the frictional forces between brackets and archwire with three passive self-ligating brackets. Eur J Orthod. 2009;31:643-6.

33. Ardeshna AP. Clinical evaluation of fiber-reinforced-plastic bonded orthodontic retainers. Am J Orthod Dentofacial Orthop. 2011 Jun;139(6):761-7.

34. Patcas R, Pedroli G. A bonding technique for fixed maxillary retainers. J Orthod. 2012 Dec;39(4):317-22.

35. Lie Sam Foek DJ, Ozcan M, Verkerke GJ, Sandham A, Dijkstra PU. Survival of flexible, braided, bonded stainless steel lingual retainers: a historic cohort study. Eur J Orthod. 2008;30:199-204.

36. Deliperi S, Bardwell DN. Reconstruction of nonvital teeth using direct fiber-reinforced composite resin: a pilot clinical study. J Adhes Dent. 2009;11:71-78.

37. Eminkahyagil N, Erkut S. An innovative approach to chairside provisional replacement of an extracted anterior tooth: use of fiber-reinforced ribbon-composites and a natural tooth. J Prosthodont. 2006;15:316-320.

38. Miller TE, Hakimzadeh F, Rudo DN. Immediate and indirect woven polyethylene ribbon-reinforced periodontal-prosthetic splint: a case report. Quintessence Int. 1995; 26: 267-271.

39. Piovesan EM, Demarco FF, Cenci MS, Pereira-Cenci T. Survival rates of endodontically treated teeth restored with fiberreinforced custom posts and cores: a 97-month study. Int $\mathrm{J}$ Prosthodont. 2007;20:633-639.

40. Turker SB, Alkumru HN, Evren B. Prospective clinical trial of polyethylene fiber ribbon-reinforced, resin composite postcore buildup restorations. Int J Prosthodont. 2007;20:55-56.

41. Van Heumen CC, van Dijken JW, Tanner J, Pikaar R, Lassila LV, Creugers NH, Vallittu PK, Kreulen CM. Five-year survival of 3-unit fiber-reinforced composite fixed partial dentures in the anterior area. Dent Mater. 2009;25:820-827.

42. Van Wijlen P. A modified technique for direct, fibre-reinforced, resin-bonded bridges: clinical case reports. J Can Dent Assoc. 2000;66:367-371.

43. Wolff D, Schach C, Kraus T, Ding P, Pritsch M, Mente J, Joerss D, Staehle HJ. Fiber-reinforced Composite Fixed Dental Prostheses: A Retrospective Clinical Examination. J Adhes Dent. 2010.

44. Brauchli L, Pintus S, Steineck M, Luthy H, Wichelhaus A. Shear modulus of 5 flowable composites to the Ever Stick Ortho fiber-reinforced composite retainer: an in-vitro study. Am J Orthod Dentofacial Orthop. 2009;135:54-58.

45. Foek DL, Ozcan M, Krebs E, Sandham A. Adhesive properties of bonded orthodontic retainers to enamel: stainless steel wire vs fiber-reinforced composites. J Adhes Dent. 2009;11:381-390.

46. Rudo DN. Clinical comparison of a multistranded wire and a direct-bonded polyethylene-reinforced resin composite used for lingual retention. Quintessence Int. 2004;35:348; author reply 349 .

47. Winkler D. Clinical comparison of a multistranded wire and a direct-bonded polyethylene-reinforced resin composite used for lingual retention. Quintessence Int. 2004;35:348; author reply 349.

48. Rudo DN, Karbhari VM. Physical behaviors of fiber reinforcement as applied to tooth stabilization. Dent Clin North Am. 1999;43:7-35.

49. Audenino G, Giannella G, Morello GM, Ceccarelli M, Carossa S, Bassi F. Resin-bonded fixed partial dentures: tenyear follow-up. Int J Prosthodont. 2006;19:22-23.

50. Kaji A, Sekino S, Ito H, Numabe Y. Influence of a mandibular fixed orthodontic retainer on periodontal health. Aust Orthod J. 2013 May;29(1):76-85.

51. Jones ML. Clinical assessment of the wider span palatal adhesive retainer. J Clin Orthod. 1987;21:740-742. 